\title{
F-index of bridge and chain graphs
}

\author{
Nilanjan De \\ Calcutta Institute of Engineering and Management, Department of Basic Sciences and Humanities, Kolkata, West Bengal, India
}

*Corresponding author: de.nilanjan@rediffmail.com

Article history

Received 22 May 2016

Accepted 1 Sept 2016

\begin{abstract}
The F-index or "Forgotten topological index" of a molecular graph is defined as the sum of cubes of degrees of all the vertices of the graph. In this paper, we compute the F-index of bridge and chain graphs. Further using these derived results, the F-index of several classes of chemical graphs and nanostructures are computed.
\end{abstract}

Keywords: Topological index, vertex degree, bridge and chain graphs, graph operations

\section{INTRODUCTION}

A molecular topological index is a numeric parameter which characterizes the topology of a molecular graph and is necessarily an invariant under automorphism of graphs. In chemical graph theory, a molecular graph is the graphical representation of the structural formula of a chemical compound whose atoms are represented using vertices and the edges represent the chemical bonds between the atoms. Topological indices correlate the physico-chemical properties of the molecular graph and have been found to be useful in isomer discrimination, quantitative structure-activity relationship (QSAR) and structure-property relationship (QSPR). Topological indices have shown its high applicability in chemistry, biochemistry and nanotechnology including discovery and design of new drugs.

In this paper, we are concerned with simple connected graphs, having no directed or weighted edges. Let $G$ be such a graph with vertex set $V(G)$ and edge set $E(G)$. Let the number of vertices and edges of $G$ will be denoted by $n$ and $m$ respectively. Also let the edge connecting the vertices $u$ and $v$ is denoted by $u v$. The degree of a vertex $v$, is the number of first neighbors of $v$ and is denoted by $d_{G}(v)$

. The Zagreb indices, first introduced by Gutman and Trinajstić [1], are most important topological indices in study of structure property correlation of molecules and have received attention in mathematical as well as chemical literature. The First and Second Zagreb Index of a graph are defined as

$$
\begin{aligned}
M_{1}(G)= & \sum_{v \in V(G)} d_{G}(u)^{2}=\sum_{u v \in E(G)}\left[d_{G}(u)+d_{G}(v)\right] \text { and } \\
& M_{2}(G)=\sum_{u, v \in V(G)} d_{G}(u) d_{G}(v)
\end{aligned}
$$

These two Zagreb indices are among the oldest molecular structure descriptors and have been extensively studied both with respect to mathematical and chemical point of view (see [2-7]).

Recently, the concept of F-index or 'forgotten topological index' attracting much attention of researchers though this index was introduced in the same paper where the first and second Zagreb indices were introduced. Furtula and Gutman in [8] recently investigated this index and showed that the predictive ability of this index is almost similar to that of first Zagreb index and for the entropy and acetic factor, both of them yield correlation coefficients greater than 0.95 . They named this index as "forgotten topological index" or "F-index". Very recently the present author studied this index for different graph operations [9] and also introduced its coindex version in [10]. De et al. in [11] and [12] also studied F-index of several classes of nanostar dendrimers and total transformation graphs. In [13], Furtula et al. explore some basic properties and bounds of Findex and in [14] Abdoa et al. investigate the trees extremal with respect to the F-index. Gao et al. in [15] present the F-index of several chemical structure which often appear in drug molecular graph. The F-index of a graph $G$ is defined as

$$
F(G)=\sum_{v \in V(G)} d_{G}(u)^{3}=\sum_{u v \in E(G)}\left[d_{G}(u)^{2}+d_{G}(v)^{2}\right]
$$

Since several classes of chemical graphs and nanostructures can be considered as a special case of bridge and chain graphs, so in mathematical chemistry it is important to study different topological indices of bridge and chain graphs, including when its components are mutually isomorphic. Already different topological indices of bridge and chain graphs have been studied. Mansour et al. in [16] and [17] calculated vertex PI index, Szeged index, Wiener, hyper-Wiener, detour and hyper-detour indices of different bridge and chain graphs. Azari et al. in [18] found some explicit expression of first and second Zagreb indices of bridge and chain graphs. 
In this paper, we continue the previous work to determine the F-index of these bridge and chain graphs. Also considering some special cases, we calculate several classes of chemical graphs and nanostructures when the components of these graphs are mutually isomorphic. Throughout this paper, as usual $C_{n}$ and $P_{n}$ denote the cycle and path graphs on $n$ vertices. In this paper, our notations are standard and mainly taken from [18].

\section{MAIN RESULTS}

In this section first we define two types of bridge graphs namely

$$
\begin{aligned}
& B_{1}=B_{1}\left(G_{1}, G_{2}, \ldots, G_{d} ; v_{1}, v_{2}, \ldots, v_{d}\right) \\
& B_{2}=B_{2}\left(G_{1}, G_{2}, \ldots, G_{d} ; v_{1}, w_{1}, v_{2}, w_{2}, \ldots, v_{d}, w_{d}\right)
\end{aligned}
$$

and hence compute their F-index. Then we define a chain graph denoted by

$$
C=C\left(G_{1}, G_{2}, \ldots, G_{d} ; v_{1}, w_{1}, v_{2}, w_{2}, \ldots, v_{d}, w_{d}\right)
$$

and hence calculate F-index of this chain graph.

\section{F-index of Bridge Graph}

The bridge graph of $\left\{G_{i}\right\}_{i=1}^{d}$ with respect to the vertices $\left\{v_{i}\right\}_{i=1}^{d}$ is denoted by

$$
B_{1}=B_{1}\left(G_{1}, G_{2}, \ldots, G_{d} ; v_{1}, v_{2}, \ldots, v_{d}\right)
$$

which is the graph obtained from $G_{1}, G_{2}, \ldots, G_{d}$ by connecting the vertices $v_{i}$ and $v_{i+1}$ by an edge for all $i=1,2, \ldots, d-1$. The bridge graph $B_{1}$ is shown in the Figure 1 . From definition of $B_{1}$ we get following Lemma directly.

Lemma 1. The degree of the vertices of $B_{1}$ is given by

$$
d_{B_{1}}(u)= \begin{cases}d_{G_{i}}(u), & \text { if } u \in V\left(G_{i}\right)-\left\{v_{i}\right\} \\ v_{1}+1, & \text { if } u=v_{1} \\ v_{i}+2, & \text { if } u=v_{i}, 2 \leq i \leq d-1 \\ v_{d}+1, & \text { if } u=v_{d}\end{cases}
$$

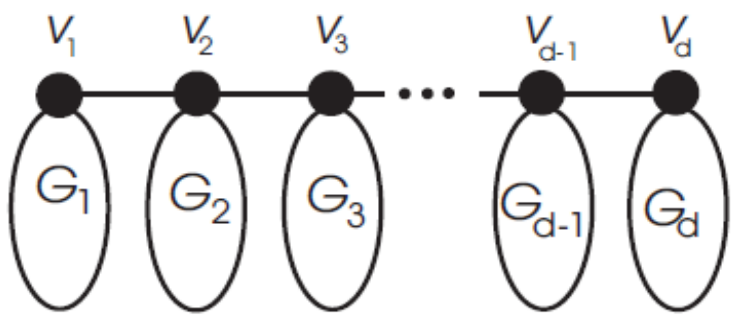

where $v_{i}=d_{G_{i}}\left(v_{i}\right)$, for $1 \leq i \leq d$.

Figure 1. The bridge graph $B_{1}=B_{1}\left(G_{1}, G_{2}, \ldots, G_{d} ; v_{1}, v_{2}, \ldots, v_{d}\right)$

Theorem 1. The F-index of the bridge graph $B_{1}, d \geq 2$, is given by

$$
\begin{aligned}
F\left(B_{1}\right)= & \sum_{i=1}^{d} F\left(G_{i}\right)+6 \sum_{i=2}^{d-1} v_{i}^{2}+12 \sum_{i=2}^{d-1} v_{i}+3\left(v_{1}^{2}+v_{d}^{2}\right. \\
& \left.+v_{1}+v_{d}\right)+8 d-14
\end{aligned}
$$

where $v_{i}=d_{G_{i}}\left(v_{i}\right)$, for $1 \leq i \leq d$.

Proof. Using definition of F-index and Lemma 1, we get

$$
\begin{aligned}
F\left(B_{1}\right)= & \sum_{i=1}^{d} \sum_{u \in V\left(G_{i}\right)-\left\{v_{i}\right\}} d_{G_{i}}(u)^{3}+\left(v_{1}+1\right)^{3}+\sum_{i=2}^{d-1}\left(v_{i}+2\right)^{3} \\
& +\left(v_{d}+1\right)^{3} \\
= & \sum_{i=1}^{d} F\left(G_{i}\right)-\sum_{i=1}^{d} v_{i}^{3}+\left(v_{1}^{3}+3 v_{1}^{2}+3 v_{1}+1\right) \\
& +\sum_{i=2}^{d-1}\left(v_{i}^{3}+6 v_{i}^{2}+12 v_{i}+8\right)+v_{d}^{3}+3 v_{d}^{2}+3 v_{d}+1 \\
= & \sum_{i=1}^{d} F\left(G_{i}\right)-\sum_{i=1}^{d} v_{i}^{3}+\sum_{i=1}^{d} v_{i}^{3}+6 \sum_{i=2}^{d-1} v_{i}^{2}+12 \sum_{i=2}^{d-1} v_{i} \\
& +3\left(v_{1}^{2}+v_{1}+v_{d}^{2}+v_{d}\right)+8 d-14
\end{aligned}
$$

from where the desired result follows.

Let, $v$ is a vertex of a graph $G$, and $G_{i}=G$ and $v_{i}=v$ for $i=1,2, \ldots, d$, then using above theorem we directly get the following :

Corollary 1. The F-index of the bridge graph $B_{1}=$ $B_{1}(G, G, ., G ; v, v, ., v), d \geq 2$, is given by

$$
F\left(B_{1}\right)=d F(G)+6(d-1) v^{2}+6(2 d-3) v+8 d-14
$$

where $v=d_{G}(v)$.

Another kind of bridge graph of $\left\{G_{i}\right\}_{i=1}^{d}$ with respect to the vertices $\left\{v_{i}, w_{i}\right\}_{i=1}^{d}$ is denoted by

$$
B_{2}=B_{2}\left(G_{1}, G_{2}, ., G_{d} ; v_{1}, w_{1}, v_{2}, w_{2}, ., v_{d}, w_{d}\right),
$$

which is the graph obtained from $G_{1}, G_{2}, \ldots, G_{d}$ by connecting the vertices $w_{i}$ and $v_{i+1}$ by an edge for all $i=1,2, \ldots, d-1$. The bridge graph $B_{2}$ is shown in the Figure 2. Similarly the following Lemma is the direct consequence of the definition of $B_{2}$.

Lemma 2. The degree of the vertices of $B_{2}$ are given by

$$
d_{B_{2}}(u)= \begin{cases}d_{G_{1}}(u), & \text { if } u \in V\left(G_{1}\right)-\left\{w_{1}\right\} \\ d_{G_{d}}(u), & \text { if } u \in V\left(G_{d}\right)-\left\{v_{d}\right\} \\ d_{G_{i}}(u), & \text { if } u \in V\left(G_{i}\right)-\left\{v_{i}, w_{i}\right\}, 2 \leq i \leq d-1 \\ w_{i}+1, & \text { if } u=w_{i}, 1 \leq i \leq d-1 \\ v_{i}+1, & \text { if } u=v_{i}, 2 \leq i \leq d\end{cases}
$$

where $v_{i}=d_{G_{i}}\left(v_{i}\right), w_{i}=d_{G_{i}}\left(w_{i}\right)$, for $1 \leq i \leq d$.

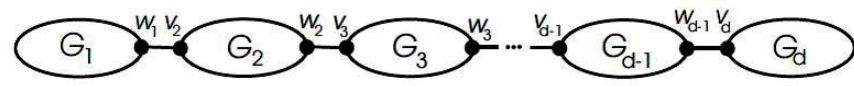

Figure 2. The bridge graph $B_{2}=B_{2}\left(G_{1}, G_{2}, \ldots, G_{d} ; v_{1}, w_{1}, v_{2}, w_{2}, \ldots, v_{d}, w_{d}\right)$

Theorem 2. The F-index of the bridge graph $B_{2}, d \geq 2$, is given by

$$
\begin{aligned}
F\left(B_{2}\right)= & \sum_{i=1}^{d} F\left(G_{i}\right)+3 \sum_{i=1}^{d-1} w_{i}^{2}+3 \sum_{i=1}^{d-1} w_{i}+3 \sum_{i=2}^{d} v_{i}^{2} \\
& +3 \sum_{i=2}^{d} v_{i}+2(d-1)
\end{aligned}
$$

where $v_{i}=d_{G_{i}}\left(v_{i}\right), w_{i}=d_{G_{i}}\left(w_{i}\right)$, for $1 \leq i \leq d$. 
Proof. Using definition of F-index and Lemma 2, we get

$$
\begin{aligned}
F\left(B_{2}\right)= & \sum_{u \in V\left(G_{1}\right)-\left\{w_{1}\right\}} d_{G_{i}}(u)^{3}+\sum_{i=2}^{d-1} \sum_{u \in V\left(G_{i}\right)-\left\{v_{i}, w_{i}\right\}} d_{G_{i}}(u)^{3} \\
& +\sum_{u \in V\left(G_{d}\right)-\left\{v_{d}\right\}} d_{G_{d}}(u)^{3}+\sum_{i=1}^{d-1}\left(w_{i}+1\right)^{3}+\sum_{i=2}^{d}\left(v_{i}+1\right)^{3} \\
& =F\left(G_{1}\right)-w_{1}^{3}+\sum_{i=2}^{d-1} F\left(G_{i}\right)-\sum_{i=2}^{d-1} v_{i}^{3}-\sum_{i=2}^{d-1} w_{i}^{3} \\
& +F\left(G_{d}\right)-v_{d}^{3}+\sum_{i=1}^{d-1}\left(w_{i}^{3}+3 w_{i}^{2}+3 w_{i}+1\right) \\
& +\sum_{i=2}^{d}\left(v_{i}^{3}+3 v_{i}^{2}+3 v_{i}+1\right) \\
= & \sum_{i=1}^{d} F\left(G_{i}\right)-\sum_{i=2}^{d} v_{i}^{3}-\sum_{i=1}^{d-1} w_{i}^{3}+\sum_{i=1}^{d-1} w_{i}^{3} \\
& +3 \sum_{i=1}^{d-1} w_{i}^{2}+3 \sum_{i=1}^{d-1} w_{i}+(d-1) \\
& +\sum_{i=2}^{d} v_{i}^{3}+3 \sum_{i=2}^{d-1} v_{i}^{2}+3 \sum_{i=2}^{d-1} v_{i}+(d-1)
\end{aligned}
$$

from where after simplification we get the desired result.

Let, $u$ and $v$ are two vertices of a graph $G$, and $G_{i}=G, v_{i}=v$ and $w_{i}=w$ for all $i=1,2, \ldots, d$. Then using above theorem we directly get the following.

Corollary 2. The F-index of the bridge graph $B_{2}, d \geq 2$, is given by

$$
F\left(B_{2}\right)=d F(G)+3(d-1)\left(v^{2}+w^{2}+v+w\right)+2(d-1)
$$

where $v=d_{G}(v), w=d_{G}(w)$.

\section{F-index of Chain Graph}

The chain graph $\left\{G_{i}\right\}_{i=1}^{d}$ with respect to the vertices $\left\{v_{i}, w_{i}\right\}_{i=1}^{d}$ is denoted by

$$
C=C\left(G_{1}, G_{2}, \ldots, G_{d} ; v_{1}, w_{1}, v_{2}, w_{2}, \ldots, v_{d}, w_{d}\right),
$$

which is the graph obtained from $G_{1}, G_{2}, \ldots, G_{d}$ by identifying the vertices $w_{i}$ and $v_{i+1}$ for all $i=1,2, \ldots, d-1$. The chain graph is shown in the Figure 3.

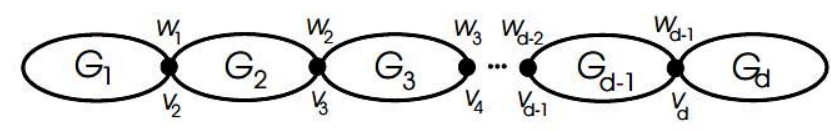

Figure 3. The chain graph $C=C\left(G_{1}, G_{2}, \ldots, G_{d} ; v_{1}, w_{1}, v_{2}, w_{2}, \ldots, v_{d}, w_{d}\right)$

From definition of chain graph $C$, we can state the following Lemma.

Lemma 3. The degree of the vertices of chain graph $C$ are given by

$$
d_{B_{1}}(v)= \begin{cases}d_{G_{1}}(v), & \text { if } v \in V\left(G_{1}\right)-\left\{w_{1}\right\} \\ d_{G_{d}}(v), & \text { if } v \in V\left(G_{d}\right)-\left\{v_{d}\right\} \\ d_{G_{i}}(v), & \text { if } v \in V\left(G_{i}\right)-\left\{v_{i}, w_{i}\right\}, 2 \leq i \leq d-1 \\ w_{i}+v_{i+1}, & \text { if } u=w_{i}=v_{i+1}, 1 \leq i \leq d-1\end{cases}
$$

where $v_{i}=d_{G_{i}}\left(v_{i}\right), w_{i}=d_{G_{i}}\left(w_{i}\right)$, for $1 \leq i \leq d$.
Theorem 3. The F-index of the Chain graph $C, d \geq 2$, is given by

$$
F(C)=\sum_{i=1}^{d} F\left(G_{i}\right)+3 \sum_{i=1}^{d-1} w_{i}{ }^{2} v_{i+1}+3 \sum_{i=1}^{d-1} w_{i} v_{i+1}^{2} .
$$

where $v_{i}=d_{G_{i}}\left(v_{i}\right), w_{i}=d_{G_{i}}\left(w_{i}\right)$, for $1 \leq i \leq d$.

Proof. Using definition of F-index and Lemma 3, we get

$$
\begin{aligned}
& F(C)= \sum_{u \in V\left(G_{1}\right)-\left\{w_{1}\right\}} d_{C}(u)^{3}+\sum_{i=2}^{d-1} \sum_{u \in V\left(G_{i}\right)-\left\{v_{i}, w_{i}\right\}} d_{C}(u)^{3} \\
&+\sum_{u \in V\left(G_{d}\right)-\left\{v_{d}\right\}} d_{C}(u)^{3}+\sum_{i=1}^{d-1} \sum_{u=w_{i}=v_{i+1}} d_{C}(u)^{3} \\
&= \sum_{u \in V\left(G_{1}\right)-\left\{w_{1}\right\}} d_{G_{1}}(u)^{3}+\sum_{i=2}^{d-1} \sum_{u \in V\left(G_{i}\right)-\left\{v_{i}, w_{i}\right\}} d_{G_{i}}(u)^{3} \\
&+\sum_{u \in V\left(G_{d}\right)-\left\{v_{d}\right\}} d_{G_{d}}(u)^{3}+\sum_{i=1}^{d-1}\left(w_{i}+v_{i+1}\right)^{3} \\
&= F\left(G_{1}\right)-w_{1}^{3}+\sum_{i=2}^{d-1} F\left(G_{i}\right)-\sum_{i=2}^{d-1} v_{i}^{3}-\sum_{i=2}^{d-1} w_{i}^{3}+F\left(G_{d}\right) \\
&-v_{d}^{3}+\sum_{i=1}^{d-1} w_{i}^{3}+\sum_{i=2}^{d} v_{i}^{3}+3 \sum_{i=1}^{d-1} w_{i}^{2} v_{i+1}+3 \sum_{i=1}^{d-1} w_{i} v_{i+1}^{2}
\end{aligned}
$$

from where the desired result follows.

Let, $u$ and $v$ are two vertices of a graph $G$, and $G_{i}=G, v_{i}=v$ and $w_{i}=w$ for $i=1,2, \ldots, d$. Then using above theorem we directly get the following.

Corollary 3. The F-index of the chain graph $C, d \geq 2$, is given by

$$
F(C)=d F(G)+3 v w(d-1)(v+w)
$$

where $v=d_{G}(v), w=d_{G}(w)$

\section{APPLICATIONS}

As an application of above derived results, in this section we compute F-index of some particular chemically interesting molecular graphs and nanostructures.

Example 1. Let us now consider a particular bridge graph, denoted by

$$
T_{d, n}=G_{d}\left(C_{n}, v\right)=B_{1}\left(C_{n}, C_{n}, \ldots, C_{n} ; v, v, \ldots, v\right)(d \text {-times }),
$$

where $v$ is an arbitrary vertex of degree 2 of $C_{n}$ (see Figure 4 for $n=6$ ). Applying Corollary 1 , after direct calculation we get the following

$$
\begin{aligned}
F\left(T_{d, n}\right) & =8 n d+24(d-1)+12(2 d-3)+6 d-14 \\
& =8 n d+56 d-74 .
\end{aligned}
$$

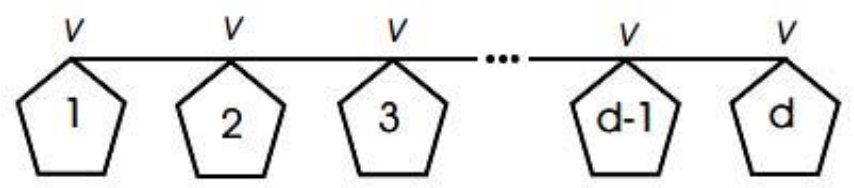

Figure 4. The bridge graph $B_{1}\left(C_{5}, C_{5}, \ldots, C_{5} ; v, v, \ldots, v\right)$

Example 2. The square comb lattice graph $C_{q}(N)$ with open ends, is the bridge graph $B_{1}\left(P_{n}, P_{n}, \ldots, P_{n} ; v, v, \ldots, v\right)$, where $N=n^{2}$ is the total 
number of vertices of $C_{q}(N)$ (see Figure 5 ). Here $P_{n}$ is the path graph and $v$ is the vertex of degree one. Thus applying Corollary 1 , we get, for $n \geq 3$

$$
\begin{aligned}
F\left(C_{q}(N)\right) & =n(8 n-14)+6(n-1)+6(2 n-3)+6 n-14 \\
& =8 n^{2}+10 n-38 .
\end{aligned}
$$

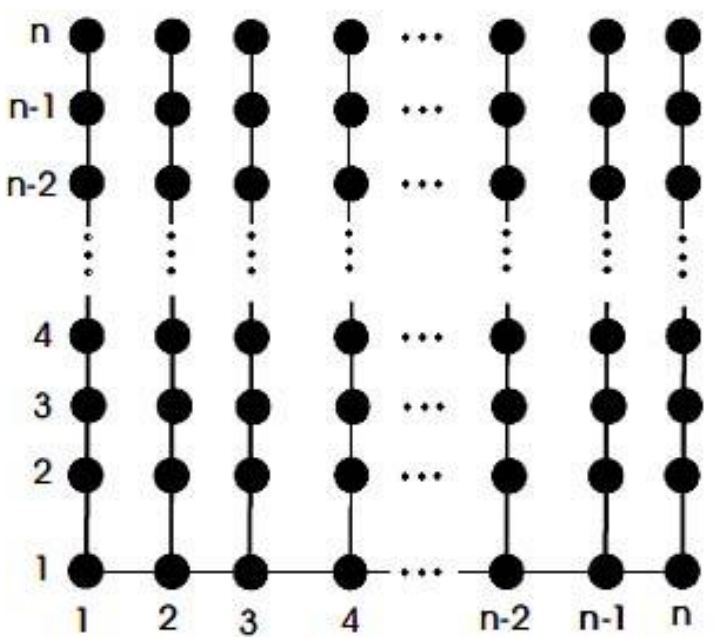

Figure 5. The square comb lattice graph $C_{q}(N)$

Example 3. Next we consider the van Hove comb lattice graph $\mathrm{CvH}(N)$ with open ends. This graph can be represented as the bridge graph $B_{1}\left(P_{1}, P_{2}, \ldots, P_{n-1}, P_{n}, P_{n-1}, \ldots, P_{2}, P_{1} ; v_{1,1}, v_{1,2}, \ldots, v_{1, n-1}, v_{1, n}, v_{1, n-1}\right.$, $\left.\ldots, v_{1,2}, v_{1,1}\right)$, where for $2 \leq i \leq n, v_{1, i}$ is the first vertex of degree one of the path graph $P_{i}$ and $v_{1,1}$ is the single vertex of degree zero of the path $P_{1}$. So, using Corollary 1 we get, after calculation

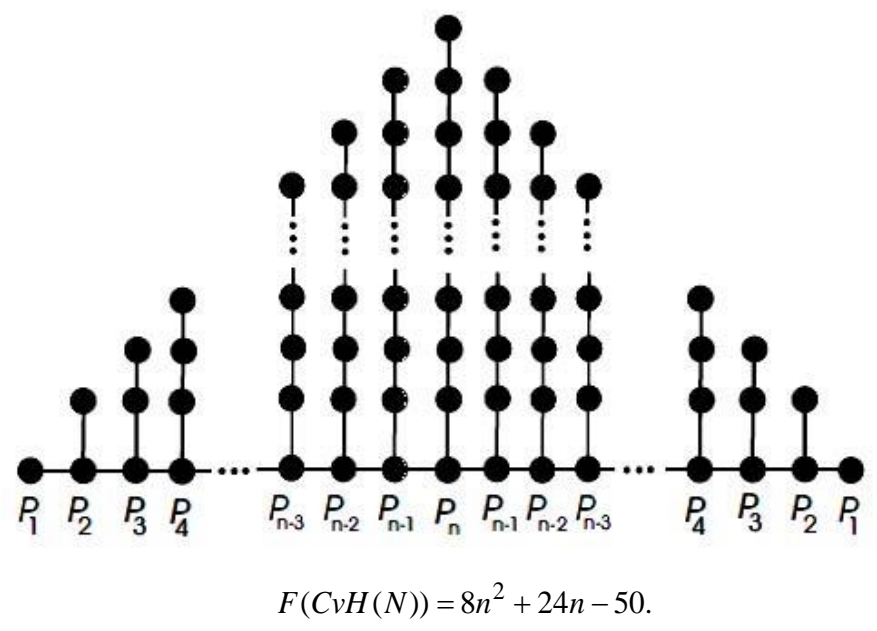

Figure 6. The van Hove comb lattice graph $\mathrm{CvH}(N)$

Example 4. The spiro-chain of the graph $G=C_{n}(k, l)(n \geq 3)$ is the chain graph $C(G, G, \ldots, G ; v, w, v, w, \ldots, v, w)$ where $k$ and $l$ are numbers of vertices $v$ and $w$ respectively. The spiro-chain of $C_{4}$ and $C_{6}$ are shown in Figure 7. The spiro-chain of $d$ number of $C_{n}(k, l)$ is denoted by $S_{d}\left(C_{n}(k, l)\right)$. Thus applying Corollary 3 , we get

$$
F\left(S_{d}\left(C_{n}(k, l)\right)\right)=8 n d+344(d-1)=8 n d+48 d-48 .
$$

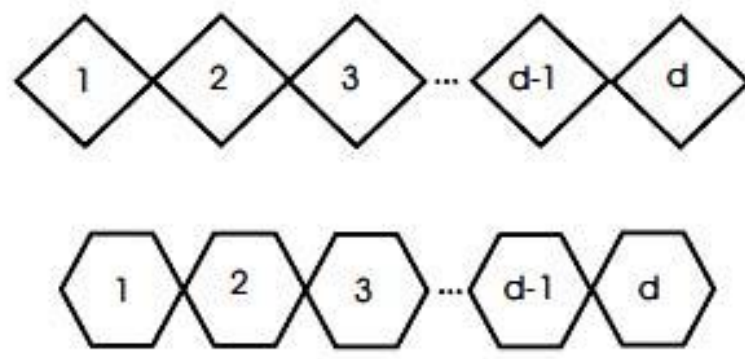

Figure 7. The spiro- chain of $C_{4}$ and $C_{6}$

Example 5. The polyphenyl chain of $h$ hexagon is said to be an ortho- $\left(\mathrm{O}_{h}\right)$, meta- $\left(\mathrm{M}_{\mathrm{h}}\right)$ and para- $\left(\mathrm{Lh}_{\mathrm{h}}\right)$, if all its internal hexagons are ortho-hexagons, meta-hexagons and para-hexagons respectively. The polyphenyl chain $\mathrm{O}_{h}, \mathrm{M}_{h}$ and $\mathrm{L}_{h}$ may be considered as the second type of bridge graph

$$
B_{2}\left(C_{6}, C_{6}, \ldots, C_{6} ; v, w, v, w, \ldots, v, w\right) .
$$

Here $C_{6}$ is the cycle graph where every vertex is of degree two (i.e. $v=w=2$ ). The ortho-, meta-, para-polyphenyl chain of hexagons is given in Figure 8. So, using Corollary 2, we get from direct calculation

$$
\begin{aligned}
F\left(O_{h}\right)= & F\left(M_{h}\right)=F\left(L_{h}\right) \\
& =48 h+3(h-1)\left(2^{2}+2^{2}+2+2\right)+2(h-1) \\
& =86 h-38 .
\end{aligned}
$$
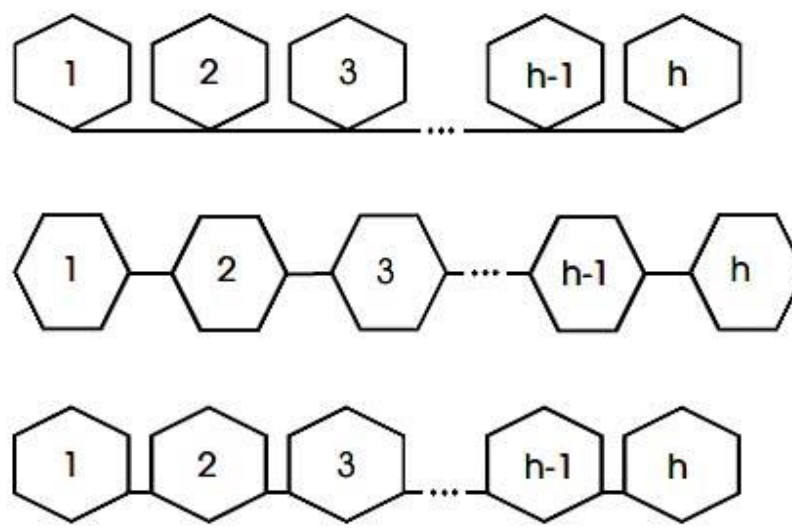

Figure 8. The ortho-, meta-, para- polyphenyl chain of hexagons

Example 6. Let us consider the molecular graph of the nanostar dendrimers $D_{n}$ (as shown in Figure 9). This graph is equivalent to the bridge graph

$$
B_{2}(G, G, \ldots, G ; v, w, v, w, \ldots, v, w) \text { ( } n \text {-times), }
$$

where $G$ is the graph given in Figure 10 and $v$ and $w$ are vertices of degree 3 and are shown in Figure 9. Thus applying corollary 2, we get

$$
\begin{aligned}
F\left(D_{n}\right) & =228 n+3(n-1)(4+4+2+2)+2(n-1) \\
& =266 n-38 .
\end{aligned}
$$




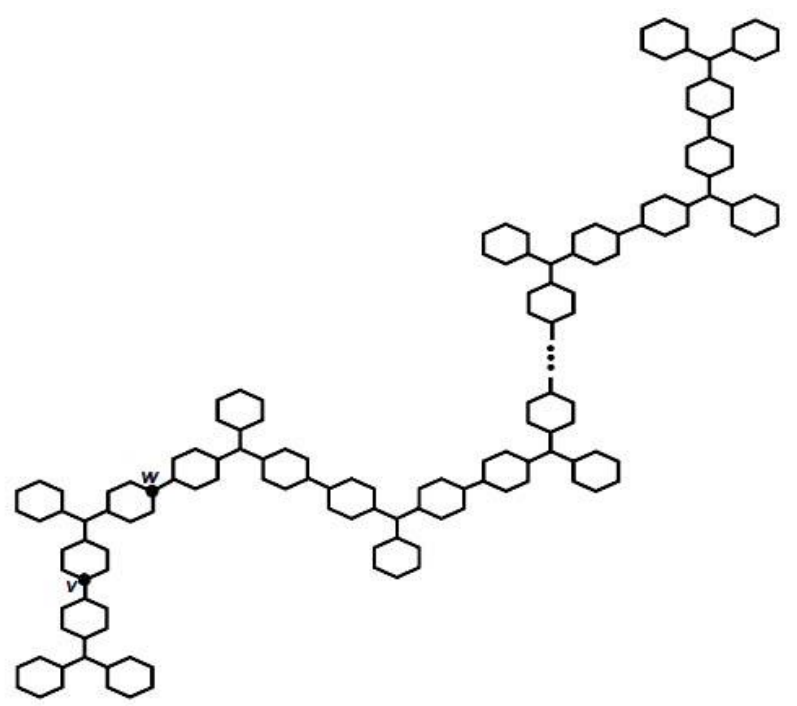

Figure 9. The molecular graph of the nanostar dendrimers $D_{n}$.

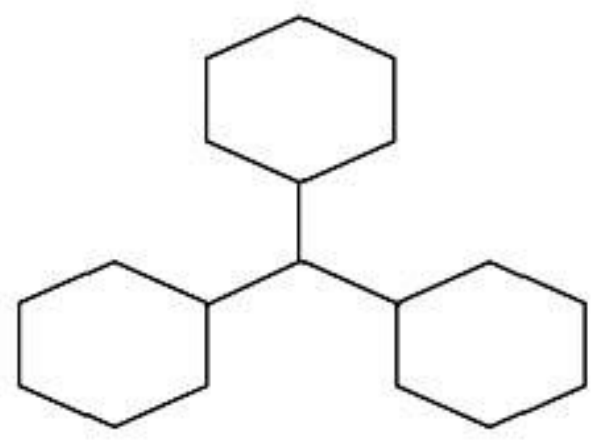

Figure 10. The molecular graph of the nanostar dendrimers $D_{1}$

\section{CONCLUSION}

In this paper, we have calculated explicit expressions of F-index for bridge and chain graphs. Furthermore as an application, in this paper we have derived expressions for several chemically important graphs and nanostructures which are considered as special cases of bridge and chain graphs, when they were built from several copies of isomorphic graphs.

\section{REFERENCES}

[1] I. Gutman, N. Trinajstić, Chem. Phys. Lett. 17, (1972), 535.

[2] K. C. Das, I. Gutman, B. Horoldagva, MATCH Commun. Math. Comput. Chem. 68, (2012), 189.

[3] M. Azari, A. Iranmanesh, MATCH Commun. Math. Comput. Chem. 70, (2013), 901.

[4] K.C. Das, K. Xu, J. Nam, Front. Math. China 10, (2015), 567.

[5] M.H. Khalifeha, H. Yousefi-Azaria, A.R. Ashrafi, Discr. Appl. Math. 157(4), (2009), 804.

[6] B. Zhou, I. Gutman, MATCH Commun. Math. Comput. Chem. 54, (2005), 233.

[7] K. Xu, K. Tang, H. Liu, J. Wang, J. Appl.Math. Inf. 33, 2015, 365.

[8] B. Furtula, I. Gutman, J. Math. Chem. 53, 2015, 1184.

[9] N. De, S.M.A. Nayeem, A. Pal, Discrete Math. Algorithms Appl. 8(2), (2016), 1650025

[10] N. De, S.M.A. Nayeem, A. Pal, SpringerPlus, 5, 2016, doi: 10.1186/s40064-016-1864-7.

[11] N. De, S.M.A. Nayeem, Pacific Science Review A: Natural Science and Engineering, doi:10.1016/j.psra.2016.06.001.

[12] N.De, arXiv:1606.05989 (preprint).

[13] B. Furtula, I. Gutman, Ž.K. Vukicevic, G. Lekishvili, G. Popivoda, Bull. Cl. Sci. Math. Nat. Sci. Math. 40, (2015), 19.

[14] H. Abdoa, D. Dimitrov, I. Gutman, arXiv:1509.03574v2 (preprint).

[15] W. Gao, M.K. Siddiqui, M. Imran, M.K. Jamil, M.R. Farahani, Saudi Pharm J. 24(3), (2016), 258.

[16]T. Mansour, M. Schork, Discr. Appl. Math. 157, (2009), 1600

[17] T. Mansour, M. Schork, J. Math. Chem. 581, (2009), 59.

[18] M. Azari, A. Iranmanesh, I. Gutman, MATCH Commun. Math. Comput. Chem. 70, (2013), 921. 